\title{
Comparison Between the Effectiveness of Mental Practice and Physical Practice using Cup Stacking on Hand Function in the Elderly
}

\author{
Jaruwan Prasomsri, PT, M.Sc. ${ }^{1}$, Jenjira Wadbanjerd, PT, B.Sc. ${ }^{2}$, Tiriporn Suttinon, PT, B.Sc. ${ }^{3}$, \\ Sakongkorn Keereena, PT, B.Sc. ${ }^{4}$ \\ 'Physical Therapy Department, Faculty of Medicine, Prince of Songkla University, Hat Yai, Songkhla 90110, Thailand. \\ ${ }^{2}$ Physical Therapy Department, Sichon Hospital, Sichon, Nakhon Si Thammarat 80120, Thailand. \\ ${ }^{3}$ Physical Therapy Department, Kantang Hospital, Kantang, Trang 92110, Thailand. \\ ${ }^{4}$ Physical Therapy Department, Phuket Provincial Hospital, Mueang, Phuket 83000, Thailand. \\ Received 17 November 2020 • Revised 2 February 2021 • Accepted 8 February 2021 • Published online 11 May 2021
}

\begin{abstract}
:
Objective: The objective of this study was to investigate the effect of mental vs. physical cup stacking practice in healthy people aged over 65 years.

Material and Methods: Healthy elderly, who met the inclusion criteria, were randomized into 2 groups-physical practice and mental practice. Both groups received cup stacking training according to the specific condition of the group. This study collected data on the immediate effects of the training using the Jebsen Hand Function Test (JHFT) for both hands, the dominant and the non-dominant ones. The movement time during cup stacking and reaction time were measured. The paired t-test and the independent t-test were selected to analyze the differences within and between groups, respectively. Results: There were 39 participants in each group. It was found that the movement time related to stacking cups and the JHFT movement time of the non-dominant hand was significantly lower in the physical practice group compared with the mental practice group. Besides, after training, the movement time for stacking cups and the JHFT movement time of both hands was significantly lower in both groups. However, a statistically nonsignificant result for reaction time was found in the mental practice group after training.
\end{abstract}

Conclusion: This study shows that both mental and physical training can enhance the ability to learn hand movement in the elderly. In further studies, the inclusion of the retention of training effects after practice, a different training approach, and a feedback method should be considered.

Keywords: elderly, hand function, mental practice, physical practice, stacking cups

Contact: Jaruwan Prasomsri, PT, M.Sc.

Physical Therapy Department, Faculty of Medicine, Prince of Songkla University, Hat Yai, Songkhla 90110, Thailand.

E-mail: jaruwan.pr@psu.ac.th

(c) 2021 JHSMR. Hosting by Prince of Songkla University. All rights reserved.

This is an open access article under the CC BY-NC-ND license

(http://www.jhsmr.org/index.php/jhsmr/about/editorialPolicies\#openAccessPolicy).
J Health Sci Med Res 2021;39(6):481-489 doi: 10.31584/jhsmr.2021809 www.jhsmr.org 


\section{Introduction}

Nowadays, the number of elderly people in Thailand is increasing. The Act on the Elderly defines the elderly as people who are older than sixty years of age. The National Statistical Office of Thailand has reported a trend of between 5.8 and $15.3 \%$ of Thai elderly experiencing disability in the past decade. ${ }^{1}$ Most activities in daily life like dressing, lifting and holding things, eating, etc., are dependent on hand and upper extremity functions, so they are a major factor in the performance of such activities by the elderly. ${ }^{2}$ Most hand functions require the movements of both hands together. Without bilateral coordination, the performance of such functions appears to be clumsy or insufficient. Thus, the function of both hands should be considered. Carmeli et al. reported that, especially after 65 years of age, the hand function decreases in both sexes. ${ }^{3}$ In turn, these changes in performance lead to a decrease in the level of the quality of life in the elderly. ${ }^{4}$ Previous studies have reported that the increasing age has an impact on brain structure, reducing the amount of both grey and white matter as well as brain function. ${ }^{5-7}$ The most affected area is the white matter in the frontal brain areas that control the body's movement. ${ }^{5-7}$ This area of the brain can process movement faster and better after manual practice and even mental practice. ${ }^{8}$

Stacking cups is a recent option for enhancing physical performance. Melanie et al. reported that stacking cups can stimulate brain function in both hemispheres and coordination between eyes and hands. Furthermore, patients can perform better in terms of both fine movements and concentration. ${ }^{9}$ Over the past decade, mental rehabilitation has gained acceptance as a new treatment method for the elderly. Motor imagery (MI) is a type of mental practice where no motor execution is seen. ${ }^{10}$ Positive results have been seen after the repeated imagery practice of the task. ${ }^{11}$ This method reduces the cost of treatment and is both safe and readily available in motor function rehabilitation. ${ }^{12}$ Gentili et al. studied movement learning through physical and imagery methods by comparing dominant with nondominant hands. ${ }^{13}$ They found that movement imagery led to the reduction of movement time and a higher precision during reaching in both hands, similarly to the results obtained after physical practice. Arnaud et al. reported that imagery practice by repeating slow movements could improve physical function in healthy elderly people. ${ }^{12}$ Thus, the findings of previous studies can be applied to elderly people with physical function problems.

However, the latest studies have reported on the effects of mental practice combined with conventional treatment on physical performance in a variety of age ranges and conditions. Yet, to the authors' knowledge, no studiy has reported clearly on the impact of only mental practice or motor imagery on physical performance among the elderly. Previous studies on motor imagery in the elderly have focused on lower extremity function, balance, and gait. Surprisingly, even though hand function is very important for the performance of activities in daily life, upper extremity and/or hand function have been largely ignored. Therefore, this study aimed to investigate the effect of mental and physical practice using stacking cups on hand function in healthy people aged over 65 years. The Jebsen Hand Function Test (JHFT), which tests the hand functions required to perfrom activities of daily living, was employed in this research.We hypothesized that the healthy elderly who receive mental practice perform better on stacking cups than those who receive physical practice.

\section{Material and Methods}

This study is an experimental research. Healthy elderly individuals aged 65 years and older were recruited. According to the sample size calculation, 39 elderly subjects are needed per group, mental practice and physical practice groups. All participants gave a written informed consent. The experimental protocol was approved by the Ethics Committee for Human Research, Faculty of Medicine, Prince 
of Songkla University on February 15, 2018 (Certificate No. REC 60-435-30-2). This study was done at Laboratory 3, Physical Therapy Department, Faculty of Medicine, Prince of Songkla University, Songkhla, Thailand. In this study, the researcher who conducted all the tests was the same person, and the researchers who trained the participants in each group were adjudged and blinded to the patients' group allocation. An inter-rater reliability test was done before data collection; a high level of reliability was observed $(r>0.9, p$-value<0.050) between the tester and the experienced user of JHFT as well as between the tester and the computer program for assessing reaction time. The participants' level of independence concerning daily activities was measured using the Barthel Index for Activities of Daily Living. Cognitive impairment was assessed via the Thai Mini-Mental State Examination. Depressive symptoms were detected through the Patient Health Qestionnaire-2 and -9 tools. In this study, the Kinesthetic and Visual Imagery Questionnaire-10 (KVIQ-10) was used to evaluate the capacity of individuals to represent different movements mentally. The standard test for fine and gross motor hand function, the JTHFT, was selected to provide an objective measurement of hand function. This study collected data regarding the immediate effects of JHFT for both hands-the dominant and non-dominant ones. The movement time during cup stacking was measured from the moment the participant lifted his/her hand over the timer until after they placed their hand on the timer again; this was detected using the New Pro Timer - G4 (TIM 008). The 3-6-3 pattern, a cup stacking pattern, was used in this study. The reaction time of the upper extremities was measured via the Red Light-Green Light Reaction Time Test. The cup stacking and reaction times were averaged from 3 and 5 repetitions, respectively. The researcher asked the participant to perform the tasks in the JHFT, cup stacking, and reaction time assessments as fast as possible.
Elderly people who were independent in performing activities of daily living (i.e., a score of least 12 on the the Barthel Index for Activities of Daily Living), had the capability to imagine movements (i.e., a score of at least 25 on KVIQ10 ), and without any depressive symptoms were included in this research. Subjects were excluded if they presented with visual impairment (not correctable with contact lens or glasses), hand sensation deficit ( $<3 / 5$ of sensory testing), significant cognitive impairment, neuromuscular disorders or musculoskeletal diseases affecting upper extremities, uncontrolled hypertension or diabetes mellitus, and unstable angina.

The healthy elderly who met the inclusion criteria were randomized into either of 2 groups-physical practice and mental practice-using simple random sampling (without replacement). Both groups received cup stacking training according to the specific condition of the group. All of the participants received information about the whole process of this study prior to signing the informed consent and the researcher starting the baseline data collection. The JHFT, reaction, and cup stacking times were measured to serve as baseline data in both groups. Before measuring the cup stacking time, the researcher demonstrated the procedure of cup stacking according to the 3-6-3 pattern to the participant and allowed them to practice 3 times. While practicing, if the participant performed the wrong pattern, the researcher would correct and give feedback to the participant immediately. After a 2-minute break, the participant in the physical practice group was told to practice stacking cups in the 3-6-3 pattern by themselves for 30 minutes while hearing the instructions for the pefromance of that procedure. However, the number of practice repetitions was left up to the participant, and the researcher recorded the number of practice runs. For the mental practice group, the participants were not allowed to perform any physical movement; they practiced by imagination only. Even though the groups received feedback differently, Sanders et al. 
found no differences in effect on study outcomes after physical and motor imagery practice. ${ }^{14}$ After they practiced for the alloted time, their cup stacking, JHFT, and reaction times were assessed. Participants had a 10-second break after each round of cup stacking testing and a 2-minute break after each test was completed.

The sample size of this study was calculated following the equation for a difference in means (equalsized groups) presented in the study of Tretriluxana et al. ${ }^{15}$ The Shapiro-Wilk Normality Test was used to analyze the distribution of data. The data related to baseline characteristics, age, and time variables (cup stacking, JHFT, and reaction times) were analyzed using descriptive statistics and the independent t-test, respectively. Moreover, the paired t-test and the independent t-test were selected to analyze the differences within and between groups, respectively. Statistical significance was set at a p-value of $<0.050$.

\section{Results}

This study recruited 85 participants, who satisfied the inclusion criteria. However, only 78 participants completed the practice and testing. The 7 participants, who did not follow the training protocol, were excluded from the study. Female sex and right-hand dominance were predominant in both groups. The mental practice and physical practice groups consisted of $64.1 \%$ and $76.9 \%$ female participants, respectively. As presented in Table 1, the baseline data did not show any significant difference between the groups.

Movement times related to cup stacking, JHFT times of the dominant and non-dominant hands, and the reaction times of both groups are presented in terms of mean and standard deviation values. The cup stacking movement times and the JHFT times for both the nondominant and dominant hands decreased significantly after mental as well as physical practice. In addition, reaction time after training was statistically non-significant in the MI group, but in the physical practice group, statistical significance was reached ( $p-v a l u e<0.050)$. The differences between mental and physical practice were statistically meaningful only regarding cup stacking movement time.

Table 2 shows the comparison of these variables within and between groups.

Table 1 Baseline charecteristics

\begin{tabular}{|c|c|c|c|}
\hline Variables & $\begin{array}{l}\text { Mental practice group } \\
(n=39)\end{array}$ & $\begin{array}{l}\text { Physical practice group } \\
(n=39)\end{array}$ & p-value \\
\hline $\mathrm{Age}^{\mathrm{a}}$ (years) & $70.92 \pm 4.66$ & $70.79 \pm 3.87$ & 0.895 \\
\hline Sex: female ${ }^{b}$ & $25(64.1 \%)$ & $30(76.9 \%)$ & 0.217 \\
\hline Dominant: right ${ }^{b}$ & $34(87.2 \%)$ & $36(92.3 \%)$ & 0.458 \\
\hline Movement time: pre-test $^{a}$ (sec) & $27.03 \pm 7.69$ & $25.94 \pm 8.91$ & 0.565 \\
\hline JHFT movement time: dominant, pre-test $^{\mathrm{a}}$ (sec) & $90.80 \pm 43.05$ & $93.70 \pm 44.64$ & 0.771 \\
\hline JHFT movement time: non-dominant, pre-test ${ }^{\mathrm{a}}$ (sec) & $120.29 \pm 54.51$ & $124.43 \pm 53.21$ & 0.735 \\
\hline Reaction time: pre-test $^{\mathrm{a}}$ (sec) & $0.42 \pm 0.14$ & $0.42 \pm 0.15$ & 0.807 \\
\hline Amount of practice ${ }^{a}$ (times) & $18.03 \pm 16.19$ & $19.77 \pm 16.12$ & 0.637 \\
\hline
\end{tabular}

$\mathrm{a}=$ mean \pm S.D. analyzed via the independent $\mathrm{t}$-test, $\mathrm{b}=$ numeral (percentage) analyzed via the chi-square test, sec=second, JHFT=Jebsen Hand Function Test

${ }^{*} \mathrm{p}-$ value $<0.050$ 
Table 2 Comparison of movement time (cup stacking), Jebsen Hand Function Test time, and reaction time within and between groups

\begin{tabular}{|c|c|c|c|c|c|}
\hline \multirow{2}{*}{ Variables } & \multicolumn{2}{|c|}{ Mental practice group $(n=39)$} & \multicolumn{2}{|c|}{ Physical practice group $(n=39)$} & \multirow{2}{*}{ p-value } \\
\hline & Pre-training & Post-training & Pre-training & Post-training & \\
\hline Movement time (sec) & $27.03 \pm 7.69$ & $21.80 \pm 6.24$ & $25.94 \pm 8.91$ & $17.06 \pm 4.74$ & $<0.001^{\mathrm{b}^{x+* x}}$ \\
\hline p-value & \multicolumn{2}{|c|}{$<0.001^{\mathrm{a}+*+*}$} & \multicolumn{2}{|c|}{$<0.001^{\mathrm{a}^{* * * *}}$} & \\
\hline JHFT movement time: Dominant (sec) & $90.80 \pm 43.05$ & $85.28 \pm 49.97$ & $93.70 \pm 44.64$ & $84.15 \pm 47.47$ & $0.912^{b}$ \\
\hline p-value & \multicolumn{2}{|c|}{$0.046^{a^{*}}$} & \multicolumn{2}{|c|}{$<0.001^{\mathrm{a}^{*+*}}$} & \\
\hline JHFT movement time: Non-dominant (sec) & $120.29 \pm 54.51$ & $104.71 \pm 44.59$ & $124.43 \pm 53.21$ & $108.24 \pm 47.47$ & $0.736^{\mathrm{b}}$ \\
\hline p-value & \multicolumn{2}{|c|}{$<0.001^{* * * *}$} & \multicolumn{2}{|c|}{$<0.001^{\mathrm{a}^{* * * *}}$} & \\
\hline Reaction time (sec) & $0.42 \pm 0.14$ & $0.38 \pm 0.07$ & $0.42 \pm 0.15$ & $0.39 \pm 0.09$ & $0.757^{b}$ \\
\hline p-value & \multicolumn{2}{|c|}{$0.097^{\mathrm{a}}$} & \multicolumn{2}{|c|}{$0.023^{\mathrm{a}^{*}}$} & \\
\hline
\end{tabular}

mean \pm S.D. analyzed using a=paired t-test, $b=$ independent $t$-test, sec=second, JHFT=Jebsen Hand Function Test ${ }^{*} \mathrm{p}$-value $<0.050,{ }^{* *} \mathrm{p}$-value $<0.010,{ }^{* \star *} \mathrm{p}-$ value $<0.005,{ }^{* \star * *} \mathrm{p}-$ value $<0.000$

\section{Discussion}

This study indicates that mental and physical training can enhance motor performance. The reduction of movement time after physical training is a direct effect of motor learning. Repeated practice helps speed up and organize signal transmission through the corticospinal tract to the target muscle and joint. ${ }^{16}$ Physical training has applied Schmid's Schema Theory in order to both plan and modify movement performance. Our results indicated a more accurate pattern and a movement time reduction after both physical and mental practice. Therefore, it can be concluded that, in this study, while learning a new movement, i.e., stacking cups, our participants may have applied the principles of this theory.

In this study, mental practice, where only imagery practice was involved to the exclusion of physical movement, yielded similar results after training as did physical practice. Arnaud et al. investigated the effects of imagery practice in healthy elderly people and found that repetitive imaginary practice can improve movement performance. ${ }^{17}$ Concordantly, we found that the cup stacking movement time decreased after motor imagery. During Ml training, the participants processed all of the sensory information about the movement that is required to perform the real movement. In this study, the sensory information was voice commands related to cup stacking instructions. The mechanism of movement control in this case started with the auditory information being sent to the brain to stimulate the motor execution. ${ }^{16}$ The effect of Ml usually occurs after the repeated imagery of a specific movement. ${ }^{5,17}$

Furthermore, we found that, in the physical practice group, movement time dropped significantly more than in the mental practice group. However, in both groups, movement time after practice declined significantly compared to the one before testing. Physical practice promotes the reduction of movement time and improves motor execution performance more than imagery practice alone. This finding is consistent with that of the study by Coffman et al., who found that the combination of physical and mental practice in healthy subjects was associated with shorter movement times compared with mental practice alone. ${ }^{18}$ Therefore, frequent physical practice leads to a proper motor performance in a given environment along with a proper mapping of the perceptual-motor workspace. ${ }^{19}$ Nevertheless, mental 
practice, like motor imagery, does not result in a greater reduction of movement time than physical practice. Motor imagery is a learning process that belongs to the cognitive stage, which is the first stage of the motor learning process. During it, learners try to understand the aim of the movement and pay attention to thinking about what they have to move. Higher brain functions control one's mind, attention, determination, and concentration required for basic leaning and movement. ${ }^{19}$

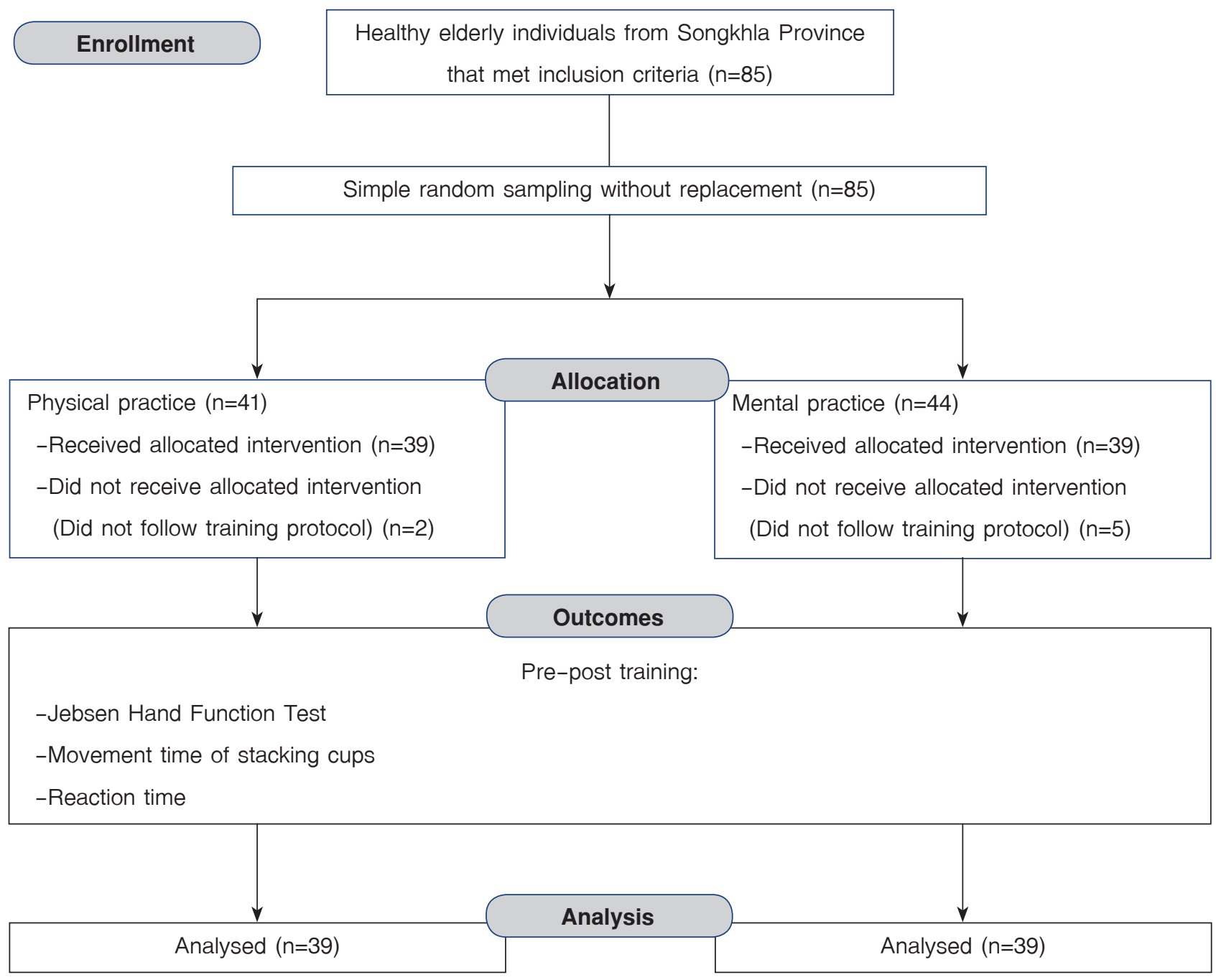

Figure 1 Consort flow diagram 
We found that JHFT movement time concerning both hands and groups decreased statistically significantly after practice ( $p$-value<0.001). The cup stacking task obviously required eye and hand coordination the same way tasks in JHFT do. Although the JHFT tasks are not exactly the same as those involved in cup stacking, the transfer task ability that persisted after both types of practice played a role in the reduction of JHFT movement times in both groups. It can be concluded that the task transferal skill led to better hand performance in this study. Therefore, both practice conditions allowed the participant to practice continuously on their own while applying the principles of the motor learning theory. ${ }^{19}$ The JHFT movement times for both hands decreased after mental practice, which concurs with the findings of the study by Muller et al. They also found that JHFT movement times in stroke patients dropped after receiving mental sequential movement practice for their fingers. ${ }^{19}$ Therefore, a continuous brain stimulation like motor imagery results in the enhancement of both movement learning and sequencing, which includes task skill retention as well as transferal from the cup stacking to the JHFT tasks, leading to faster JHFT movement times.

As was the case with the mental practice group, the physical practice group saw lower JHFT movement times in both hands after practice. This finding is consistent with those of the study by Friedhelm et al. on the effect of 30 minutes of physical practice on hand functions. They found that statistically significantly faster hand movement times and better hand function performance compared with before training. ${ }^{19}$ Hand movement time is reduced after physical practice as a result of the movement learning process. This process recruits information from sounds, somatosensory information, and the proprioception sense, which is processed during movement. ${ }^{19}$ In addition, the task skill transferal from the cup stacking practice to the JHFT tasks causes the shortening of JHFT movement times.
When comparing the two types of practice, we found that the JHFT movement times of both hands decreased after practice, albeit not significantly. However, there was a significant decrease after practice compared with before practice in both conditions. This study found that mental practice encourages motor learning in both hands as much as physical practice does. Furthermore, after mental practice, in the mental practice group, the non-dominant hand showed a stronger decreasing trend in movement times than the dominant hand. Therefore, it can be said that mental practice may be better than physical practice at promoting motor learning in the non-dominant hand. This finding supports those of the Liu et al. 2014 study, which looked at functional magnetic resonance imaging results after mental practice in acute stroke patients and found that mental practice yielded greater brain activity corresponding to the affected hand than in the unaffected one. ${ }^{20}$ The reason why mental practice enhances hand movement in the nondominant hand may be associated with the performance plateau phenomenon. ${ }^{19}$ Therefore, we can conclude that mental practice may be useful and appropriate for task training when participants have no experience with the task, such as training for the non-dominant hand or patients who have lost the ability to control their movements, in order to regain their movement performance.

Reaction time after practice in this study presented a non-significant difference in the mental group and between groups. The study by Alikhan et al. found that choice reaction time showed signs of difference after motor imagery practice for 2 weeks in healthy young adults. ${ }^{21}$ However, this study saw no difference after practice in the mental group, and this may have been due to the inappropriate tool used to measure reaction time or the insufficient practice time allotted. A previous study suggested that the appropriate tool for measuring reaction time should employ choice reaction time; it should not be a simple one like the one used in 
this study. ${ }^{21}$ Choice reaction time revealed a higher level of task complexity compared with simple reaction time. The pre-movement phase of reaction response relies on the learner's decisions, so mental practice may help shorten the time required for this phase. Hence, choice reaction time may be a more appropriate tool for measuring reaction time after motor imagery training. However, since in this study we measured the immediate effect of training, perhaps the training intensity was not enough to yield an observable difference. Consequently, we may have been unable to detect any difference after the practice sessions.

Conversely, the physical practice group showed significantly lower reaction times after practice ( $p$-value 0.023). Physical practice helps cut the time used in the movement-time phase. The study by Abourezk et al. found that, after physical exercise for 5 years, simple reaction time in healthy elderly individuals showed no meaningful difference. ${ }^{22}$ Fatemeh et al. reported that, after physical practice for 8 weeks in moderately cognitively-impaired elderly women using a computer program designed for brain training, a significant decrease in simple reaction time was observed. ${ }^{23}$ Similarly, Light et al. found that, after 160 repetitions of arm-reaching movement practice for 2 days in healthy elderly subjects, the reaction time measured on the day after training was significantly different between the study groups. ${ }^{24}$ Because in this study we measured the immediate effect, maybe our training intensity was insufficient to lead to observable differences. Moreover, since both groups showed decreased reaction times after practice, it is possible that differences between groups were present but not large enough to be detected by our assessment tools.

\section{Conclusion}

In this study, immediately after cup stacking practice according to individual pace and number of repetitions, it was found that movement time and JHFT time in both hands decreased significantly following physical and mental practice. Physical practice showed a significantly bigger movement time improvement compared to mental practice as well as a greater reduction in reaction time after practice only in the physical practice group. However, we found no significant difference between groups in terms of JHFT movement time in both hands and reaction time. These findings demonstrate that both physical and mental practice can enhance the motor learning of hand functions in healthy elderly people. Mental practice should be adopted in training or rehabilitation programs in our routine practice in order to increase the effectiveness of motor learning training, especially among patients who are unable to move during the early stages of the disease, e.g., stroke patients. This study investigated only the immediate effects of mental and physical practice using cup stacking tasks in terms of hand functions, so the retention effect after practice remains unknown. Furthermore, the training approaches between the two groups and the way the participants received feedback were different. In further studies, adding complex choice reaction time to measure reaction time along with the study of the non-dominant hand are recommended. Moreover, including the retention effect after practice, different training approaches, and feedback methods should be considered by future research.

\section{Acknowledgement}

We would like to express our deep gratitude to all participants in this study for their co-operation. We would also like to extend our thanks to the International Affairs Office, Faculty of Medicine, Prince of Songkla University for their help with editing this manuscript.

\section{Funding source}

This study was supported by a scholarship from the Faculty of Medicine, Prince of Songkla University. 


\section{Conflict of interest}

There are no potential conflicts of interest to declare.

\section{References}

1. Dop.go.th. Older statistics [homepage on the Internet] Bangkok: Department of Older Persons [cited 2019 Oct 13]. Available from: http://www.dop.go.th/th/know/1

2. Maes C, Gooijers J, Orban de Xivry JJ, Swinnen SP, Boisgontier MP. Two hands, one brain, and aging. Neurosci Biobehav Rev 2017;75:234-56.

3. Carmeli E, Patish H, Coleman R. The aging hand.J Gerontol Series A 2003;58:146-52.

4. Miyawaki T, Kumamoto K, Shimoda K, Tozato F, Iwaya T. Relationship among motor function, ADL disability, and psychological concerns in elderly people with locomotive disorders. J Orthop Sci 2017;22:339-44.

5. Giorgio A, Santelli L, Tomassini V, Bosnell R, Smith S, De Stefano $\mathrm{N}$, et al. Age-related changes in grey and white matter structure throughout adulthood. Neurolmage 2010;51: 943-51.

6. Sullivan EV, Pfefferbaum A. Neuroradiological characterization of normal adult ageing. Br J Radiol 2007;80:S99-108.

7. Salat $\mathrm{DH}$. The declining infrastructure of the aging brain. Brain Connec 2011;1:279-93.

8. Bloom F, Beal F, Kupfer D. The Dana Guide to Brain Health New York: Simon \& Schuster; 2003.

9. Hart MA, Bixby WR. Brain activation patterns during participation in cup stacking. RQES 2005;76:A57.

10. Jeannerod M. Mental imagery in the motor context. Neuropsychologia 1995;33:1419-32.

11. Feltz DL, Landers DM. The effects of mental practice on moto skill learning and performance: a meta-analysis. J Sport Exerc Psychol 1983;5:25-57.

12. Saimpont A, Malouin F, Tousignant B, Jackson PL. Motor imagery and aging. J Mot Behav 2013;45:21-8.
13. Gentili RJ, Papaxanthis C. Laterality effects in motor learning by mental practice in right-handers. Neuron 2015;297:231-42.

14. Sanders C, Sadoski M, Bramson R, Wiprud R, Walsum K. Comparing the effects of physical practice and mental imagery rehearsal on learning basic skills by medical students. AJOG 2004;191:1811-4.

15. Tretriluxana J, Taptong J, Chaiyawat P. Dyad training protocol on learning of bimanual cup stacking in individuals with stroke: effects of observation duration. J Med Assoc Thai 2015;98:S106-12.

16. Cameron MH, Monroe L. Physical rehabilitation: evidencebased examination, evaluation, and intervention. St. Louis: Elsevier Health Sciences; 2007.

17. Saimpont A, Malouin F, Tousignant B, Jackson PL. Motor imagery and aging. J Mot Behav 2013;45:21-8.

18. Coffman DD. Effects of mental practice, physical practice, and knowledge of results on piano performance. J Res Music Educ 1990;38:187-96.

19. Shumway-Cook A, Woollacott MH. Motor control: translating research into clinical practice. Maryland: Lippincott Williams \& Wilkins; 2007

20. Liu H, Song LP, Zhang T. Mental practice combined with physical practice to enhance hand recovery in stroke patients. Behav Neurol 2014;2014:876416.

21. Alikhani $H$, VaezMousavi M, Mokhtari P. The effect of cognitive and motivational imagery on choice reaction time. World Appl Sci J 2011;12:792-6.

22. Abourezk T, Toole T. Effect of task complexity on the relationship between physical fitness and reaction time in older women. J Aging Phys Act 1995;3:251-60.

23. Husseini $F$, Damirchi A, Babaei P. Effect of brain training on cognitive performance in elderly women diagnosed with mild cognitive impairment. Caspian J Neurol Sci 2016;2:25-31.

24. Light KE, Reilly MA, Behrman AL, Spirduso WW. Reaction times and movement times: benefits of practice to younger and older adults. J Aging Phys Act 1996;4:27-41. 